\title{
Sozialgeschichtliche Aspekte des Steinkohlenbergbaus im Lugau-Oelsnitzer Revier
}

\author{
von \\ SVEN WERNER
}

Der industrielle Steinkohlenbergbau in Sachsen blieb - gemessen an der langen Bergbaugeschichte des Landes - ein historisch gesehen relativ kurzes Phänomen, ${ }^{1}$ er hat die industrielle Entwicklung Mitteldeutschlands jedoch nachhaltig forciert. Das Lugau-Oelsnitzer Revier lieferte über einen Zeitraum von ca. 130 Jahren rund 142 Millionen Tonnen Steinkohle. Wenn die Förder- und Belegschaftszahlen deutschlandweit betrachtet auch vergleichsweise gering waren ${ }^{2}$ und der sächsische Steinkohlenbergbau selbst unter Hinzuziehung des älteren und reicheren Zwickauer Kohlenausbringens zu keiner Zeit die Bedeutung des westfälischen oder oberschlesischen Steinkohlenbergbaus erreichte, ${ }^{3}$ ist insbesondere die Bedeutung als Rohstofflieferant für die Industrialisierung der mittel- und westsächsischen Region nicht zu unterschätzen. Auch über 40 Jahre nach der Einstellung der Kohlenförderung im Lugau-Oelsnitzer Revier ist die Kultur der Region noch immer stark vom Bergbau und seinen Traditionen geprägt, was sich unter anderem in einem starken Bezug auf die Montanregion Erzgebirge und in den verbreiteten Hinweisen auf die bergbauliche Geschichte in Form von Industriedenkmälern, Bergbaulehrpfaden und in der überregional bekannten Institution des Bergbaumuseums in Oelsnitz/Erzgebirge zeigt.

Der nachfolgende Aufsatz richtet sich an bergbauhistorisch interessierte Personen und fokussiert als Überblick über einen eng umgrenzten Teilbereich des sächsischen Bergbaus ausdrücklich auf das Lugau-Oelsnitzer Revier, da an dieser Stelle die anderen bedeutenden sächsischen Abbaugebiete, wie Freital und vor

1 Im Zwickauer und im Hainichener Gebiet wurde schon seit mehreren Hundert Jahren nach Kohlen gegraben; vgl. Waldemar May/August Eckhard, Die Entwicklung des Steinkohlenbergbaus im erzgebirgischen Becken, Zwickau 1938. Der hier in Rede stehende industrielle Steinkohlenbergbau in Lugau und in Oelsnitz/Erzgebirge ist historisch gesehen jedoch relativ „neu“.

2 Vgl. Rolf Vogel, Das Lugau-Oelsnitzer Steinkohlenrevier, Hohenstein-Ernstthal 1992, S. 6.

3 Der sächsische Steinkohlenbergbau trug zur Gesamtförderung des Deutschen Reichs um 1900 nur etwa 4,4 \% bei; vgl. Andreas Erb/Mona Harring (Bearb.), Aus der Tiefe ans Licht. Bildmaterialien zur Geschichte des sächsischen Steinkohlenbergbaues, Halle/ Saale 2006, S. 9. Am Anfang der 1930er-Jahre betrug beispielsweise die Gesamtmenge des Kohlenausbringens im sächsischen Steinkohlenbergbau 3.564.108 t im Wert von 71.823.418 RM; vgl. Glückauf. Berg- und hüttenmännische Zeitschrift 67 (1931), 2, S. B $44 \mathrm{f}$. 
allem Zwickau, nicht dargestellt werden können. Es wird kein Anspruch auf vollständige Wiedergabe des Quellen- und Forschungsstandes erhoben in der Weise, wie sie das unter der Leitung von Klaus Tenfelde begonnene Projekt zur Darstellung der deutschen Bergbaugeschichte leistet. ${ }^{4}$ Der historische Überblick zur Entwicklung des Reviers erfolgt insbesondere unter Verweis auf die Arbeiten von Pabst 19285, Richter $1936^{6}$ und 19407, May/Stutzer/Eckhardt $1936^{8}$ und May/ Eckhardt 19389, Fritzsche 194910, Vogel $1992^{11}$ und Erb/Harring 200612. Auch wenn im Hinblick auf soziale Begleitumstände des Steinkohlenbergbaus überblickhaft verschiedene Problemlagen benannt werden, sind die zahlreichen positiven Begleitumstände der Erschließung und Entwicklung des Lugau-Oelsnitzer Reviers unvergessen - wie etwa die Modernisierung der Infrastruktur, die kulturelle und wirtschaftliche Bereicherung der vormals stark agrarisch geprägten Region ${ }^{13}$ sowie die Möglichkeit des Einkommenserwerbs für breite Bevölkerungsteile.

\section{Zum Beginn des Koblenabbaus im Lugau-Oelsnitzer Revier}

Der als „erzgebirgisches Becken“ bekannte westliche Teil des Erzgebirgsvorlandes wurde unmittelbar vor der Aufnahme des Steinkohlenbergbaus neben dem weit verbreiteten landwirtschaftlichen Erwerb von "textilem Hausgewerbe“ und von ersten Manufakturen und Fabriken dominiert. ${ }^{14}$ Nach den ersten größeren Kohlenfunden des Zwickauer Maschinenmeisters Karl Gottlob Wolf im Grenzgebiet

4 Klaus Tenfelde/Stefan Berger/Hans-Christoph Seidel, Geschichte des deutschen Bergbaus, Bd. 1: Der alteuropäische Abbau von den Anfängen bis zur Mitte des 18. Jahrhunderts, Münster 2012.

5 Hans Pabst, Entwicklung und Absatzverhältnisse des sächsischen Steinkohlenbergbaus unter besonderer Berücksichtigung der Gegenwart, Univ. Diss. Würzburg 1928.

6 Johannes Richter, Allerlei zur Heimatgeschichte des Lugau-Oelsnitzer Kohlenbezirkes, Lugau 1936.

7 Johannes Richter, Die Entwicklung der Kulturlandschaft im Steinkohlenrevier Lugau-Oelsnitz (Erzgebirge), Univ. Diss. Jena 1940.

8 Waldemar May/Otto Stutzer/August Eckhard, 75 Jahre Gemeinschaftsarbeit der Sächsischen Steinkohlenbergwerke, Zwickau 1936.

9 Waldemar May/August Eckhard, Die Entwicklung des Steinkohlenbergbaues im erzgebirgischen Becken, Zwickau 1938.

10 Hellmut Fritzsche, Lehrbuch der Bergbaukunde mit besonderer Berücksichtigung des Steinkohlenbergbaues, 1. Band, Berlin/Göttingen/Heidelberg 81949.

11 Vogel, Steinkohlenrevier (wie Anm. 2).

12 Erb/Harring, Aus der Tiefe ans Licht (wie Anm. 3).

13 Vgl. Wolfgang Frech/Emil Haubold, Lugau/Erzgebirge. Erinnerungen an vergangene Tage, Horb am Neckar 1993, S. 7.

14 Vgl. Horst Rössler/Dietrich ZüHlke (Hg.), Zwischen Mülsengrund, Stollberg und Zwönitztal. Ergebnisse der heimatkundlichen Bestandsaufnahme in den Gebieten von Lichtenstein, Oelsnitz, Stollberg und Thalheim, Berlin 1981, S. 20. 
zwischen (Neu)Oelsnitz und Niederwürschnitz und nach erfolgreichen oberflächennahen Abbauversuchen in den 1840er-Jahren trat aufgrund der nun zunehmend oberflächenfernen Lagerung der kohlenführenden Schichten, die eine herkömm-liche Förderung mit Haspel und Förderkörben nicht mehr erlaubten, eine (vor allem technisch und finanziell bedingte) „Stagnation“ der Kleinbetriebe ein, die im Wesentlichen durch die Beschaffung größerer finanzieller und technischer Betriebsmittel und durch den Zusammenschluss in größeren Gesellschaften - wie beispielsweise im Jahre 1855 mit der Gründung des Zwickau-Lugauer Steinkohlenbauvereins - beendet werden konnte. Nach Horst Rößler und Dietrich Zühlke war die erste Förderphase durch sogenannte Eigenlöhner und durch kleine Fördergesellschaften charakterisiert, ${ }^{15}$ der Förderschwerpunkt lag auf dem Gebiet des heutigen Ortsteils Neuoelsnitz und verlagerte sich bald in Richtung Lugau.

$\mathrm{Ab} 1856$ wurde die erste moderne Schachtförderanlage im Revier - die „Neue Fundgrube“ - abgeteuft, ${ }^{16}$ die dem o. e. Zwickau-Lugauer Steinkohlenbauverein gehörte und die im Sommer 1867 Schauplatz einer international beachteten Katastrophe wurde, als ein Schachtbruch über 100 Bergleute verschüttete, die nicht gerettet werden konnten.

Im gleichen Jahr wurde auch der Steinkohlenbauverein „Gottes Segen“ gegründet und auf Lugauer Gebiet in räumlicher Nähe zur oben erwähnten Fundgrube mit dem Abteufen des Gottes-Segen-Schachtes begonnen. Die ebenfalls 1856 gegründete Oelsnitzer Bergbaugesellschaft legte 1857 mit dem Hedwigschacht die Grundlage für die Steinkohlenförderung in Oelsnitz, ${ }^{17}$ und als vier Jahre nach der 1858 erfolgten Inbetriebnahme der Bahnverbindung Niederwürschnitz-Wüstenbrand der Zwickau-Lugauer Steinkohlenbauverein im Jahr „1862 [...] einen Gleisanschluss an die Chemnitz-Würschnitzer Kohlenbahn" erhielt, ${ }^{18}$ begann damit ein weiteres entscheidendes Kapitel der Geschichte des Steinkohlenbergbaus im Lugau-Oelsnitzer Gebiet.

Der Schwerpunkt der Kohlenförderung hatte sich, wie schon erwähnt wurde, von Neuoelsnitz und Niederwürschnitz in Richtung Lugau verlagert, da sich einerseits die Niederwürschnitzer und Neuoelsnitzer Vorkommen rasch erschöpften und andererseits die technische Entwicklung nun stärkere (zum Beispiel dampfgetriebene) Fördermaschinen, eine effektivere Wasserhaltung und Bewetterung und den Abbau oberflächenferner Flöze in Gersdorf und Lugau ermöglichte. ${ }^{19}$ Nach 1871 erfolgte ein Gründungsboom bei den Steinkohlenbetrieben, von denen sich zur Kapitalbeschaffung viele zu größeren Aktiengesellschaften organisierten. Der Steinkohlenbauverein Deutschland - der Vorläufer der später bedeu-

15 Vgl. ebd., S. 142.

16 Vgl. Erb/Harring, Aus der Tiefe ans Licht (wie Anm. 3), S. 22.

17 Vgl. ebd., S. 23.

18 Ebd., S. 22.

19 Vgl. Rössler/ZüHLke, Mülsengrund (wie Anm. 14), S. 142. 
tenden Gewerkschaft Deutschland - wurde gegründet ${ }^{20}$ und das Netz der Zechenbahnen erfuhr einen Ausbau.

\section{Problematische Lebens- und Arbeitsbedingungen in der früberen und mittleren Phase der Förderung}

Die eben erwähnte Rationalisierung und Technisierung des Steinkohlenbergbaus war jedoch nicht nur mit Modernisierungseffekten verbunden, sondern hatte auch problematische Lebens- und Arbeitsbedingungen im Gefolge, die nachfolgend an drei Problemclustern exemplarisch benannt werden: erstens gesundheitliche Gefahren unter und über Tage, zweitens die Wohnungsnot und die hohen Lebenshaltungskosten und drittens die starke Fluktuation im Personalbestand der Kohlenbetriebe und der Arbeitskampf im Lugau-Oelsnitzer Revier.

\section{Gesundheitliche Gefahren unter und über Tage}

In der Phase des oben erwähnten technischen und wirtschaftlichen Aufschwungs im Steinkohlenbergbau waren die Lebens- und Arbeitsbedingungen der angelegten Bergbauarbeiter häufig als problematisch zu bezeichnen. Auf einer ersten Analyseebene kann sehr vereinfachend von "Gefahren für Leib und Leben“ gesprochen werden, die der Steinkohlenbergbau für die Angelegten und ihre Familien mit sich brachte. Rößler/Zühlke referieren hierzu Erhebungen eines Oelsnitzer Arztes Michaelis „[d]emzufolge [...] in der Mitte des 19. Jahrhunderts das Durchschnittsalter des niedererzgebirgischen Industrie- und Landproletariats 12 Jahre und 3 Monate [betrug], [...] die Kindersterblichkeit (bis zum 5. Lebensjahr) $70 \%$ [erreichte] und [...] jeder Einwohner, der das 12. Lebensjahr vollendet hatte, nur mit einer Lebenserwartung von 26 Jahren rechnen [konnte]“. ${ }^{21}$ Im Besonderen gab es eine Reihe typischer Erkrankungen, denen die Bergbaubeschäftigten ausgesetzt waren. Hierzu gehörten nach Menzel vor allem rheumatische Erkrankungen, Bandscheibenschäden und Erkrankungen des Bewegungsapparates, obstruktive Erkrankungen der Atemwege, Staublungenerkrankungen (Anthrakose, Silikose und andere), Lungentuberkulose, Herz-Kreislauferkrankungen, Augenzittern oder Nystagmus, Hauterkrankungen (Krätze, „Hüttenkrätze“, „Pechakne“, degenerative Erkrankungen und Tumore), Schwielen, Furunkel, Pilzerkrankungen, Infektionen, Hakenwurmerkrankungen (Ankylostomiasis), Blutarmut (Anämie) und Vergiftungen. ${ }^{22}$ Es erscheint nahe liegend, Ursachen dieser weit verbreiteten Symptome unter Tage in der schlecht beleuchteten Arbeitssituation, der häufig gebückten Haltung, dem Kontakt zu potenziell giftigen

20 Vgl. Erb/Harring, Aus der Tiefe ans Licht (wie Anm. 3), S. 26.

21 Vgl. Rössler/ZüHlke, Mülsengrund (wie Anm. 14), S. 21.

22 Vgl. Elmar Menzel, Bergbau-Medizin einst und jetzt. Entwicklung des bergmännischen Gesundheitswesens unter Einschluß der Kranken- und Unfallversicherung, Berlin 1989. 
Substanzen und in den nicht ungefährlichen Ventilationsverhältnissen zu suchen. Über Tage wirkten neben der Luftbelastung durch Abgase und Ruß die aufgrund des geringen Verdienstes häufig einseitige Ernährung und die beengten Wohnverhältnisse mit den entsprechenden hygienischen Problemen (und häufig noch durch sogenannte Kostgänger verschärft) auf die Bergbaubeschäftigten und ihre Familien ein.

Eine Vielzahl von Unfällen aufgrund des sicherheitstechnisch mangelhaften Ausbaus der Schächte oder des Anlegens von Bergfremden gehörten ebenfalls zum Alltag vieler Arbeiter im Revier. Besonders schockierend (und im Nachgang politisierend) dürften in diesem Zusammenhang die Bergwerksunglücke mit zahlreichen Toten und Verletzten - und hier besonders die schon erwähnte Katastrophe von 1867 auf der Neuen Fundgrube mit über einhundert Todesopfern - gewirkt haben. ${ }^{23}$

Abgesehen von derartigen Großereignissen ${ }^{24}$ wurde deutschlandweit auch nach den sogenannten Bismarckschen Sozialversicherungsgesetzen der 1880er-Jahre die soziale Fürsorge für die Bergwerksbeschäftigten sukzessive institutionalisiert und eine Reihe von betrieblichen Knappschaftsverbänden nahm die Fürsorge für kranke und verletzte Bergmänner - sowie im Todesfall für ihre hinterbliebenen Angehörigen - auf. Ab 1865 musste jeder angelegte Bergarbeiter einer regionalen Knappschaft angehören, die ab 1885 der Knappschafts-Berufsgenossenschaft in Berlin unterstand. ${ }^{25}$

23 Noch heute ist die Ursache dieses Unglücks, das vielen hiesigen Familien den Ernährer nahm, umstritten, wenngleich das Jahrbuch für den Berg- und Hütten-Mann zum Verschulden bemerkt obne irgend ein Verschulden, casus tragicus; Jahrbuch für den Berg- und Hütten-Mann auf das Jahr 1869, S. 97. Die Unterstellung, dass mangelhafter Ausbau der Strecken den Schachtbruch verursacht und menschliches Versagen unmittelbar nach dem Ereignis die schnelle Bergung der Verschütteten verhindert habe, hielt sich lange in der öffentlichen Meinung; vgl. Rössler/ZüHLke, Mülsengrund (wie Anm. 14), S. 84.

24 Vgl. Rössler/ZüHLKe, Mülsengrund (wie Anm. 14), S. 79 zur Schlagwetterexplosion auf dem Hohndorfer Idaschacht von 1884 mit 17 Todesopfern; und S. 124 zur Explosion im Oelsnitzer Friedensschacht, die 57 Menschenleben forderte.

25 Menzel, Bergbaumedizin (wie Anm. 22), S. 96. So existierten beispielsweise im Jahr des großen Fundgrubenunglücks 1867 im Lugau-Oelsnitzer Raum folgende Knappschaften: Bockwa-Oberhohndorfer Knappschaftscasse, Knappschaftscasse des Oberhohndorfer Forststeinkohlenbauvereins, Knappschaftscasse des Zwickau-Oberhohndorfer Steinkohlenbauvereins, Knappschaftscasse des Hohndorf-Bernsdorfer Steinkohlenbauvereins, Casse des Erlbach-Mittelbach-Oberlungwitzer Knappschaftsverbands, Knappschaftscasse der Fürstlich-Schönburgschen Steinkohlenwerke zu Oelsnitz, Knappschaftscasse des Sewaldschen Steinkohlenwerks zu Niederwürschnitz, Knappschaftscasse des Niederwürschnitzer Steinkohlenbauvereins, Knappschaftscasse des Lugau-Niederwürschnitzer Steinkohlenbauvereins, Knappschaftscasse des Niederwürschnitz-Kirchberger Steinkohlenactienvereins, Casse des Lugauer KnappschaftsVerbands, Knappschaftscasse des Rödlitzer Steinkohlenbauvereins und die Gemeinschaftliche Kranken- und Unterstützungscasse für Tagearbeiter, welche nicht Knappschaftsmitglieder sind, auf den zum Lugauer Knappschaftsverbande gehörigen Werken. Vgl. Jahrbuch für den Berg- und Hütten-Mann auf das Jahr 1869, S. 90-92. 
2. Wohnungsnot und hohe Lebenshaltungskosten

Die Entdeckung der Kohle und die Entwicklung der vormals agrarisch und halbindustriell geprägten kleinen Ortschaften im Revier hatte infolge der Zuwanderung Tausender Arbeitssuchender ins Revier ${ }^{26}$ - welche beispielsweise die Wohnbevölkerung von Oelsnitz, das seit dem ausgehenden 19. Jahrhundert zum Hauptabbaugebiet der Steinkohle im Revier wurde, verzehnfachte - rasch zu einer Wohnungsnot geführt. ${ }^{27}$ Die Bevölkerungszunahme in Lugau, Oelsnitz, Hohndorf und Umgebung verlief zeitlich parallel mit einer Bevölkerungszunahme in ganz Westsachsen - insbesondere im Umfeld von Zwickau, einer Stadt, die ihre Bevölkerungszahl zwischen 1871 und 1925 reichlich verdoppelte, und im Raum Chemnitz, wo sich die Bevölkerungszahl im gleichen Zeitraum mehr als verdreifachte. ${ }^{28}$

Trotz der langen und körperlich auszehrenden Schichten von bis zu 14 Stunden reichte der Lohn bei vielen dieser zugezogenen (und nicht genug Land zur Subsistenzwirtschaft besitzenden) Familien oft nicht für die Befriedigung der Grundbedürfnisse, wie eine helle, geheizte und trockene Wohnung und eine abwechslungsreiche Ernährung und die Gesundheitsfürsorge. Nach Pabst und Philipp ${ }^{29}$ mussten sich im Westsächsischen beide Seiten - sowohl die Betriebe bezüglich der Lohnentwicklung als auch die Bergarbeiterhaushalte bezüglich der Lebenshaltungskosten - an den höheren Löhnen der Industriearbeiter (und den entsprechenden Preisen) im Zwickau-Chemnitzer Industriegürtel orientieren. ${ }^{30}$ Bernhardt gibt für das Jahr 1928 im sächsischen Steinkohlenbergbau einen durchschnittlichen Jahresbruttolohn von 2.409,66 RM für einen Hauer an, wobei seine Aufstellung der Lebenshaltungskosten eines Bergarbeiterhaushalts zeigt, wie knapp mit dem verfügbaren Einkommen kalkuliert werden musste. ${ }^{31}$ Im Zusammenhang mit den in den jeweiligen „Jahrbüchern für das Berg- und Hüttenwesen

26 Vgl. Rössler/ZüHLke, Mülsengrund (wie Anm. 14), S. 75, 187 f.

27 „Schmucklose Wohnhäuser, aber auch Behelfsunterkünfte und Baracken für auswärtige Bergarbeiter, wenige Geschäftshäuser [...] dafür zahlreiche Gaststätten und Winkelkneipen prägten mit den ursprünglich bäuerlichen Anwesen und den kleinen Häusern aus der Zeit des textilen Hausgewerbes ein uneinheitliches Siedlungsbild“; ebd., S. 120. „Zwischen 1900 und 1936 zogen nach Oelsnitz insgesamt 3175 Italiener, 1713 Tschechen und 376 Polen sowie 3426 Deutsche zu“; ebd., S. 125.

28 Siegfried Grundmann, Bevölkerungsentwicklung in Ostdeutschland. Demographische Strukturen und räumliche Wandlungsprozesse seit 1945, Opladen 1998, S. 297.

29 Gerhard Philipp, Die soziale Belastung des sächsischen Steinkohlenbergbaus unter besonderer Berücksichtigung der rechtlichen Bestimmungen über Beiträge auf Grund des Reichsknappschaftsgesetzes, Univ. Diss. Leipzig 1931. Philipp führt nicht nur Daten zur Lohnentwicklung im sächsischen Steinkohlenbergbau auf, sondern interpretiert diese und geht neben einer Darstellung lohnrelevanter sozialrechtlicher Bestimmungen auf Belastungen und Gestehungskosten in den wichtigsten sächsischen Steinkohlenbetrieben ein; vgl. ebd., S. 75 f.

30 Vgl. Pabst, Absatzverhältnisse (wie Anm. 5), S. 46.

31 Karl Bernhardt, Probleme der Lage der Bergarbeiter im sächsischen Steinkohlenbergbau in den Jahren 1933 bis 1939, Univ. Diss Halle-Wittenberg 1966, S. III/7. 
in Sachsen“ aufgeführten Beitrags- und Pensionszahlungen lassen sich weitere Aufschlüsse über die soziale Schichtung innerhalb der Steinkohlenbetriebe und über die Lohnentwicklung - und hierbei auch über die Varianz innerhalb der einzelnen Gehaltsgruppen - gewinnen.

\section{Fluktuation im Personalbestand und Arbeitskampf im Lugau-Oelsnitzer Revier}

Trotz der starken Bevölkerungszunahme nach 1870 gab es auch in Lugau, Oelsnitz und Umgebung viele Auspendler nach Chemnitz, bzw. in die heimische Textilindustrie. ${ }^{32} \mathrm{Ab} 1924 \mathrm{kam}$ es zu einer richtiggehenden „Massenabwanderung“, 33 weshalb im Zusammenhang mit der Fluktuation weniger die „puren“ Beschäftigtenzahlen, sondern vielmehr die Abkehr-Quoten eine deutliche Sprache sprechen. Besonders auffällige Rückgänge sind in den Jahren 1915, 1924 und 1925 sowie 1927 zu verzeichnen. ${ }^{34}$

\begin{tabular}{|l|l|l|}
\hline Steinkohlenwerk & Belegschaft & Abkehr (in \%) \\
\hline Morgenstern & 2.890 & $1.080(37,4)$ \\
\hline Gottes Segen & 4.193 & $\begin{array}{l}3.659(87,3) \\
\text { davon } 6,5 \% \text { durch Invalidisierung }\end{array}$ \\
\hline Deutschland & 3.687 & $3.272(87,7)$ \\
\hline Gersdorfer StKbV & 629 & $619(98,4)$ \\
\hline
\end{tabular}

Tab. 1: Zablenbeispiel für die Abkehr von Bergarbeitern im Jahr 1928 im Bereich ZwickauOelsnitz. ${ }^{35}$

Als Gegenmaßnahmen der Arbeitgeberseite gegen die massenhafte Abkehr von den Schächten wurde unter anderem ein Leistungszuschuss etabliert (welcher durch den weitgehend „gedeckelten“ Grundlohn die Steuer- und Knappschaftsabzüge und das spätere Arbeitslosengeld niedrig halten sollte) und in die Errichtung von Bergarbeiterwohnungen investiert. ${ }^{36}$ Daneben erfolgten auch verstärkt Kon-

32 Vgl. Philipp, Belastung (wie Anm. 29), S. 45 f.

33 Vgl. ebd., S. 47.

34 May/Eckhard, Entwicklung (wie Anm. 9), S. 235.

35 Quelle: Philıpe, Belastung (wie Anm. 29), S. 48.

36 Rößler/Zühlke erwähnen die Anlage von Werkssiedlungen in Gersdorf (in der Nähe von Pluto, Merkur und Kaisergrube), in Oelsnitz (zum Beispiel in der Concordiastraße) und in Lugau; vgl. Rössler/ZüHLKe, Mülsengrund (wie Anm. 14), S. 76 f. In Lugau wurden darüber hinaus ein „umfangreicher kommunaler Wohnungsbau, Förderung der Siedlungsbewegung, Lehrmittelfreiheit und Milchspeisung in den Schulen [...] sowie kostenlose Totenbestattung" realisiert; ebd., S. 86. 
trollen bei Krankschreibungen und Rücksprachen mit den Arbeitsämtern, um freiwillig abgekehrten Beschäftigten die Arbeitslosenunterstützung zu sperren. ${ }^{37}$

Als 1929/30 infolge der Wirtschaftskrise viele Unternehmen in Konkurs gingen und ihre Beschäftigten freisetzten, veränderte sich regional die Balance von Arbeitskräfteangebot und -nachfrage zugunsten der Steinkohlenwerke, und es kam zu einem Überangebot an Arbeitskräften. Die allgemein schlechte wirtschaftliche Lage und die finanzielle Bedürftigkeit bewegten nun viele Arbeiter, die in der Vergangenheit schon einmal vom Schacht abgegangen waren und den Bergbau eigentlich verlassen wollten, wieder beim Steinkohlenbergbau um eine Arbeitsstelle nachzusuchen. Die meisten dieser Gesuche wurden abschlägig beschieden, denn vom Arbeitskräftemangel der späten 1930er- und frühen 1940er-Jahre war noch nichts zu spüren. Im Gegenteil: Durch das Eindringen ausländischer Kohle in den deutschen Markt gab es Probleme, die heimische Kohle abzusetzen und die - in Sachsen besonders hohen - Selbstkosten der Steinkohlengewinnung zu decken. ${ }^{38}$

Die letzten Jahre der Weimarer Republik waren nicht nur in Sachsen, sondern deutschlandweit von einem allgemein sinkenden Lebensstandard ${ }^{39}$ und von starken, teils gewaltsamen, politischen Auseinandersetzungen geprägt. Dabei waren die jährlichen Durchschnittslöhne aller im Steinkohlenbergbau Beschäftigten um 1930 im Bergamtsbezirk Stollberg mit 2.813 RM gegenüber 2.635 RM im Bergamtsbezirk Dresden und 2.500 RM im Bergamtsbezirk Zwickau noch vergleichsweise hoch. ${ }^{40}$

Die schon erwähnten problematischen Arbeits- und Lebensumstände vieler angelegter Bergarbeiter führten - einleitend mit einem ersten reichsweiten Bergarbeiterstreik im Jahr 1889 - zu einer Vielzahl von Arbeitskämpfen und Konflikten. Im gesamten niederen Erzgebirge und Erzgebirgsvorland waren kommunistische und links-sozialdemokratische Positionen stark vertreten, und so erinnert beispielsweise eine Wandtafel im Ortszentrum von Lugau daran, dass organisierte Lugauer Bergarbeiter regelmäßig mit Karl Marx korrespondierten. Es bestanden außerdem Kontakte zu August Bebel und Wilhelm Liebknecht, und bei der Wahl zum Norddeutschen Reichstag von 1867 gingen die Wahlkreise 17 (GlauchauMeerane) und 19 (Stollberg-Schneeberg) an diese Vertreter des linken Flügels der Arbeiterbewegung. ${ }^{41}$ Nach Rößler/Zühlke führte vor allem die Fundgrubenkatastrophe in Verbindung mit dem Kontakt zu Kadern der kommunistischen Bewe-

37 Vgl. Philipp, Belastung (wie Anm. 29), S. 49; und Sächsisches Staatsarchiv - Bergarchiv Freiberg (im Folgenden: BA Freiberg), Bestand 40110 - 18.

38 Vgl. BA Freiberg, Bestand $40110-1-17$.

39 Infolge eines Schiedsspruchs zum Tarifkonflikt zwischen dem Arbeitgeberverband des Steinkohlenbergbaus und der Interessenvertretung der Arbeitnehmer trat am 8. Oktober 1931 eine Lobnkürzung von 7 \% ein. Schließlich wurden die Löbne auf Grund der 4. Verordnung des Reichspräsidenten zur Sicherung der Wirtschaft und Finanzen und zum Schutze des inneren Friedens [...] am 1. Januar 1932 um weitere $10 \%$ gekürzt; Jahrbuch für das Berg- und Hüttenwesen in Sachsen 106 (1932), S. B 108.

40 Vgl. Glückauf. Berg- und hüttenmännische Zeitschrift 67 (1931), 2, B 107.

41 Vgl. Rössler/ZüHLKe, Mülsengrund (wie Anm. 14), S. 23, 69. 
gung mittelbar „zur gewerkschaftlichen Organisation in der Internationalen Gewerksgenossenschaft der Berg- und Hüttenarbeiter, die sich der I. Internationale anschloß“.42 Für die Gemeinde Oelsnitz wird darüber hinaus eine gewerkschaftliche Infrastruktur unter anderem aus einem sozialdemokratischen Wahlverein, Sächsischem Bergarbeiterverband, Konsumverein und „proletarische[n] Sportund Kulturvereine[n] " erwähnt. ${ }^{43}$ Eine ähnliche Infrastruktur existierte in Lugau, ${ }^{44}$ wobei zu ergänzen ist, dass es sowohl in Lugau als auch in Oelsnitz ${ }^{45}$ durchaus auch deutschnationale und patriotische Vereine gab, so zum Beispiel die 1860 gegründete Schützengesellschaft ${ }^{46}$ oder die Turnerschaft Germania ${ }^{47}$ in Lugau sowie in Oelsnitz den 1858 gegründeten Königlich Sächsischen Militärverein, den 1887 gegründeten Verein reichstreuer Wähler, den Verein königstreuer Knappen im Lugau-Ölsnitzer Bergrevier von 1895 oder seit 1899 eine Ortsgruppe des Deutschen Flottenvereins. Das organisatorische Spektrum, welches auch eine gewisse politische und religiöse Vielfalt wiedergab, war also sehr differenziert, wenngleich Organisationen der sogenannten Arbeiterbewegung aufgrund ihrer Vernetzung den stärksten Einfluss hatten.

Wichtige Meilensteine in den politischen Auseinandersetzungen zwischen Arbeitgebern und Arbeitnehmern im Steinkohlenbergbau waren die Reichsversicherungsordnung von $1911,{ }^{48}$ das 1920 in Kraft getretene Betriebsrätegesetz ${ }^{49}$ und das die Trägerschaft der Knappschaftsversicherung regelnde Reichsknappschaftsgesetz von 1924 mit einer Novelle im Jahr 1926.50 In der Zeit der Weimarer Republik war die Region um Oelsnitz, Lugau, Gersdorf und Hohndorf stark von Streiks ${ }^{51}$ und politischen Auseinandersetzungen, bis hin zu Reichswehreinsätzen, betroffen, daneben war unmittelbar vor der Machtübernahme der Nationalsozialisten im Jahr 1933 im Arbeitsamtsbezirk Lugau ${ }^{52}$ die Arbeitslosigkeit mit 11.622 Personen sehr hoch. 53

$$
\text { die }
$$
Hohenstein-Ernstthal, Hohndorf, Kuhschnappel, Lichtenstein-Callnberg, Lugau/Erzgebirge, Mitteldorf, Niederdorf, Oberdorf, Neuwiese, Niederwürschnitz, Oberwürschnitz, Oberlungwitz, Oelsnitz/Erzgebirge, Pfaffenhain, Reichenbach, Rödlitz, Rüsdorf, Seifersdorf, St.-Egidien, Stollberg, Tirschheim und Ursprung; vgl. BA Freiberg, Bestand $40110-1$ - 17, Schreiben an den Bergbaulichen Verein zu Zwickau e. V. vom 30. Dezember 1930.

Ebd., S. 84.

Ebd., S. 128.

Vgl. Frech/Haubold, Erinnerungen (wie Anm. 13), S. 127 ff.

Vgl. http://www.oelsnitz.net/index.php/vereine/179.html (Zugriff: 30. November 2011).

Vgl. Frech/Haubold, Erinnerungen (wie Anm. 13), S. 129.

Vgl. ebd., S. 135.

Vgl. Menzel, Bergbaumedizin (wie Anm. 22), S. 236.

Vgl. ebd., S. 328.

Vgl. ebd., S. 342.

Vgl. Pabst, Absatzverhältnisse (wie Anm. 5), S. 44.

Dem Arbeitsamt Lugau unterstanden nach eigener Darstellung zum 30. Dezember 1930 die Ortschaften Bernsdorf, Erlbach, Gablenz, Gersdorf, Heinrichsort, Hermsdorf, Vgl. BA Freiberg, Bestand $40110-1-17$. 


\section{Forcierung der Koblenförderung im Dritten Reich, Einsatz von „Ostarbeitern" und Kriegsgefangenen}

Zum Zeitpunkt der Machtübernahme der Nationalsozialisten befand sich, wie die oben erwähnten Arbeitslosenzahlen zeigen, der sächsische Steinkohlenbergbau „in einer tiefen wirtschaftlichen Krise“.54 Die bekannten und als abbauwürdig eingestuften Kohlenvorräte gingen zur Neige und der Abbau unter ungünstigen geologischen Verhältnissen und in dem dicht besiedelten Gebiet um Zwickau und zwischen Lugau, Gersdorf und Oelsnitz verursachte hohe Folgekosten, zum Beispiel für den Versatz oder für die Bergschadenregulierung. ${ }^{55}$ Der sächsische Anteil an der Gesamtkohlenförderung im Reich ging teilweise auf 2 bis $3 \%$ zurück, was speziell im Lugau-Oelsnitzer Revier bereits zu Betriebsstilllegungen und Feierschichten geführt hatte. ${ }^{56}$ Die Steinkohlenförderung stand in Lugau kurz vor der Einstellung. Durch das Zusammentreffen dieser und noch weiterer ungünstiger Bedingungen (Kapitalknappheit und politische Blockierung als Spätfolgen der Weltwirtschaftskrise von 1929) herrschte zum Zeitpunkt der Machtübernahme generell ein Arbeitskräfteüberangebot im westsächsischen Steinkohlenbergbau und in ganz Sachsen, das die reichsweit höchste Arbeitslosenquote zu verzeichnen hatte. ${ }^{57}$

Unter der NS-Herrschaft erlebte der Steinkohlenbergbau zunächst eine Forcierung, da die absehbaren Kriegsvorbereitungen und planwirtschaftliche Elemente wie der „Vierjahresplan“ auch ökonomisch unrentable Wege autarker Rohstoffgewinnung und -verarbeitung interessant machten. Die technische und organisatorische Forcierung ging mit einem Abbau der Arbeitnehmerrechte und mit der Zerschlagung der Gewerkschaften ${ }^{58}$ einher, deren organisatorischer Nachfolger die am 10. Mai 1933 gegründete „Deutsche Arbeitsfront“ wurde. Auch blieb der Bergbau hinsichtlich der Unfallziffern und der Erkrankungsraten in den 1930er-Jahren deutlich exponiert ${ }^{59}$ und brachte der relativ hohe Organisationsgrad und die politische Orientierung vieler Bergbaubeschäftigter im Revier60 in der Frühphase des Dritten Reichs ein hohes Konfliktpotenzial mit sich.

54 Stefan Posta, Terror statt Anlernung. Zwangsarbeit im sächsischen Steinkohlenbergbau während des Zweiten Weltkrieges, in: Hans-Christoph Seidel/Klaus Tenfelde (Hg.), Zwangsarbeit im Bergwerk. Der Arbeitseinsatz im Kohlenbergbau des Deutschen Reiches und der besetzten Gebiete im Ersten und Zweiten Weltkrieg, Bd. 1: Forschungen, Essen 2005, S. 193-221, hier S. 194.

55 Vgl. ebd.

56 Vgl. ebd., S. $194 \mathrm{f}$.

57 Vgl. ebd., S. 196.

58 Gewerkschaften meint hier die Arbeitnehmervereinigungen und nicht die ebenfalls als "Gewerkschaft" bezeichneten Lugauer und Oelsnitzer Steinkohlenbetriebe. Die Zerschlagung erfolgte unter anderem durch die Notverordnung zum Schutz von Volk und Staat von 1933, das sogenannte Ermächtigungsgesetz und das Gesetz zur Ordnung der nationalen Arbeit von 1934; vgl. Menzel, Bergbaumedizin (wie Anm. 22), S. 330. Vgl. ebd., S. 274

60 Rössler/ZüHLke, Mülsengrund (wie Anm. 14), S. 128. 
In den späten 1930er-Jahren entstand mit dem „Sächsischen Steinkohlensyndikat m.b.H." erstmals eine firmenübergreifende Struktur für den westsächsischen Steinkohlenbergbau (und in gewisser Weise ein organisatorischer Vorläufer der späteren VVB Steinkohle Zwickau). Am 2. März 1939 trat Görings „Verordnung zur Erhöhung der Förderleistung und des Leistungslohnes im Bergbau" in Kraft, durch welche die (seit den Arbeitskämpfen der frühen Weimarer Jahre) normale Schichtzeit unter Tage ${ }^{61}$ von 8 auf 8 3/4 Stunden erhöht wurde. ${ }^{62}$ Gleichzeitig wurden durch die „Eisern sparen“-Kampagne und die Anlage von sogenannten Mutschmann-Siedlungen im Revier soziale Anreize geschaffen, welche die Arbeitsmotivation und die Identifikation mit dem Bergarbeiterberuf stärken sollten. ${ }^{63}$

Der Ausbruch des Zweiten Weltkrieges sollte die oben erwähnte Arbeitskräftesituation im Lugau-Oelsnitzer Revier nachhaltig ändern und generell durch den Material- und Arbeitskräftemangel (infolge von Einberufungen zur Wehrmacht, aber auch durch freiwillige Abgänge zum Heer, zur SA oder zur SS) sehr stark hemmend auf den Steinkohlenbergbau einwirken. Im Zusammenhang mit sozialen Begleitumständen des Steinkohlenbergbaus im Lugau-Oelsnitzer Revier ist darum auf eine seit den 1940er-Jahren immer bedeutsamer werdende Quelle menschlicher Arbeitskraft einzugehen - die nach Deutschland geworbenen oder abkommandierten sogenannten Ostarbeiter und die in den Bergbau gepressten (vor allem sowjetischen) Kriegsgefangenen.

Im Jahr 1941, d. h. zu Beginn des Russlandfeldzugs, war im Revier bereits ein Förderrückgang aufgrund der zur Wehrmacht einberufenen Stammbelegschaftsmitglieder zu registrieren, die nicht wie zunächst geplant durch bergbauerfahrene Arbeitskräfte aus den sogenannten Satellitenstaaten (Slowakei, Kroatien) ersetzt werden konnten. ${ }^{4}$ Beispielhaft illustriert den Förderrückgang eine Auflistung der Gesamtförderung der Gewerkschaft Gottes Segen von 1935 bis 1943:

\begin{tabular}{|l|l|}
\hline Jahr & Gesamtförderung in $\mathbf{t}$ \\
\hline 1935 & 863.130 \\
\hline 1936 & 1.008 .360 \\
\hline 1937 & 1.159 .641 \\
\hline 1938 & 1.103 .639 \\
\hline 1939 & 1.094 .228 \\
\hline 1940 & 1.128 .823 \\
\hline 1941 & 1.049 .780 \\
\hline
\end{tabular}

61 Über Tage galten längere Arbeitszeiten.

62 Vgl. Wolfgang Jäger/Klaus Tenfelde (Hg.), Bildgeschichte der deutschen Bergarbeiterbewegung, bearb. von Wolfgang Jäger, München 1989, S. 172.

63 Martin Mutschmann war der NS-Gauleiter von Sachsen.

64 Vgl. Posta, Terror (wie Anm. 54), S. 198 f. 


\begin{tabular}{|l|l|}
\hline 1942 & 940.230 \\
\hline 1943 & 934.452 \\
\hline
\end{tabular}

Tab. 2: Auflistung der Gesamtförderung der Gewerkschaft Gottes Segen von 1935 bis 1943.65

Als Reaktion auf den Belegschafts- und Förderrückgang wurde vonseiten der „Bezirksgruppe Steinkohlenbergbau Mitteldeutschland“ bei der Reichsvereinigung Kohle und bei den Reichsministerien für Wirtschaft und Arbeit die Zuweisung von Kriegsgefangenen beantragt. Ab dem Frühjahr 1942 erfolgte zunächst eine Zuweisung von 40 französischen Kriegsgefangenen an die Gewerkschaft Gottes Segen, doch verweigerte diese Gruppe zeitweise unter Bezugnahme auf das Völkerrecht die Arbeit und wurde deshalb nach wenigen Monaten wieder entlassen. Auch die kurzzeitig bei Gottes Segen eingesetzten britischen Kriegsgefangenen konnten nicht in dem Maße herangezogen werden wie später die Ostarbeiter und die russischen Kriegsgefangenen, da sie unter anderem mit dem Internationalen Roten Kreuz in Kontakt standen, das sie mit Lebensmittelpaketen versorgte, ${ }^{66}$ was eine Erpressung, wie mit der sogenannten Leistungsernährung im Fall der Ostarbeiter ${ }^{67}$ unmöglich machte. Zudem wurden vonseiten des OKW bei der Behandlung der britischen Kriegsgefangenen Opportunitätskalküle verfolgt. ${ }^{68}$

Die im Juni 1942 auf der Gewerkschaft Deutschland angelegten 75 ukrainischen Ostarbeiter erwartete ein anderes Los. ${ }^{69}$ Diese ins Dritte Reich gekommenen Freiwilligen aus der Ukraine, die zumindest theoretisch die deutschen (Ungelernten-) Tarife verdienen sollten ${ }^{70}$ waren durch die problematischen Transport- und Ernährungsverhältnisse geschwächt, häufig nicht bergbauerfahren und mit den vorgefundenen Arbeits- und Lebensbedingungen völlig überfordert. ${ }^{71}$

Diese schlechten Lebens- und Arbeitsbedingungen (zum Beispiel die Unterbringung in umzäunten Lagern mit Verbot des Ortswechsels, ${ }^{72}$ Kleidung mit dis-

65 Quelle: BA Freiberg, Bestand $40110-1-171$.

66 Vgl. Posta, Terror (wie Anm. 54), S. 215 f.

67 Vgl. ebd., S. 211; Hans-Christoph Seidel/Klaus Tenfelde (Hg.), Zwangsarbeit im Bergwerk. Der Arbeitseinsatz im Kohlenbergbau des Deutschen Reiches und der besetzten Gebiete im Ersten und Zweiten Weltkrieg, Bd. 2: Dokumente, Essen 2005, S. $409,634$.

68 Vgl. Jörg Osterloh, Die Lebensbedingungen und der Arbeitseinsatz von Kriegsgefangenen im „Dritten Reich“ und in der Sowjetunion, in: Hans-Christoph Seidel/ Klaus Tenfelde (Hg.), Zwangsarbeit im Europa des 20. Jahrhunderts. Bewältigung und vergleichende Aspekte (Schriftenreihe Arbeitseinsatz und Zwangsarbeit im Bergbau 5), Essen 2007, S. 155-186, hier S. $161 \mathrm{f}$.

69 Vgl. Posta, Terror (wie Anm. 54), S. 201 f.

70 Vgl. Seidel/Tenfelde, Zwangsarbeit (wie Anm. 67), S. 551.

71 Vgl. Posta, Terror (wie Anm. 54), S. $201 \mathrm{f}$.

72 Vgl. Seidel/Tenfelde, Zwangsarbeit (wie Anm. 67), S. 277. 
kriminierenden Abzeichen, niedriger Lohn und mangelhafte Ernährung sowie die strikte Kontrolle durch die Gestapo ${ }^{73}$ ) sorgten dafür, dass trotz eines Norm- und Strafensystems (mit zum Beispiel Lohn- und Nahrungskürzung) nur mangelhafte Arbeitsleistungen erreicht wurden. Ein Anlernen oder eine Übertragung von Verantwortung fanden so gut wie nicht statt, ${ }^{74}$ da vonseiten der Steinkohlenwerke erstens angenommen wurde, der sogenannte Blitzkrieg würde die einberufenen deutschen Belegschaftsmitglieder bald wieder freigeben, und da zweitens davon ausgegangen wurde, dass im Falle längerer Personalausfälle der deutschen Stammbelegschaft oder nach dem Ablegen bergbauuntauglicher Ostarbeiter noch viele Kriegsgefangene als „Ersatz“ bereitstünden. ${ }^{75}$ Durch die vermehrte Einberufung deutscher Belegschaftsangehöriger zur Wehrmacht kumulierte jedoch im Laufe des Jahres 1942 der errechnete Arbeitskräftebedarf in den sächsischen Steinkohlenbetrieben auf über 1.000 Personen und darüber hinaus waren die auf der Gewerkschaft Deutschland angelegten ukrainischen Arbeitskräfte - vermutlich aus Rentabilitätsgründen - inzwischen wieder entlassen worden. Die Betriebsleitungen der westsächsischen Steinkohlenwerke versuchten nun, bezugnehmend auf einen sogenannten Führerbefehl vom Herbst $1941^{76}$ den Belegschaftsrückgang durch die Aufnahme von sowjetischen Kriegsgefangenen 77 - insgesamt „etwas mehr als 1.100 sowjetische Kriegsgefangene bei den Gruben in Lugau, in Oelsnitz und Zwickau"78- zu kompensieren. Parallel zu den Bemühungen um die Zuweisung von Kriegsgefangenen wurden weiterhin Ostarbeiter angelegt, zum Beispiel eine Gruppe von 56 Personen im Frühjahr 1943 auf Gottes Segen. Während die Zivilarbeiter über die Arbeitsämter in den besetzten Gebieten zum „Reichseinsatz" vermittelt wurden, kamen die Gefangenen mehrheitlich aus den Stammlagern IV F Hartmannsdorf und IV B Mühlberg (d. h. aus der Zuständigkeit des Wehrkreiskommandos Dresden), wo sich - wie im ganzen Gebiet des Deutschen Reichs - erst ab 1942 die Ansicht durchsetzte, Kriegsgefangene von der Ostfront als Arbeitskräfte einzusetzen, was zur Folge hatte, dass es zumindest halbherzige Bemühungen gab, das Massensterben in den Lagern einzuschränken. ${ }^{79}$ Für den

73 Im Falle fortgesetzter Normuntererfüllung oder beim Verdacht der „Hetze“ wurden die Ostarbeiter durch die Betriebsleitungen der Gestapo gemeldet; vgl. zur Praxis auf Gottes Segen: ebd., S. 318f.

74 Vgl. Posta, Terror (wie Anm. 54), S. 206.

75 Vgl. ebd.

76 Vgl. Osterloh, Lebensbedingungen (wie Anm. 68), S. 168.

77 „Die sowjetischen Kriegsgefangenen waren ein wichtiges Arbeitskräftepotenzial für die deutsche Kriegswirtschaft [...] 1944 waren im Durchschnitt allein 160.000 sowjetische Kriegsgefangene im Bergbau eingesetzt [...]. Das OKW bezifferte den monatlichen ,Durchschnittsverbrauch ‘ an Gefangenen im Steinkohlenbergbau - gemeint waren Tote und Arbeitsunfähige - auf 3,3 Prozent“; Osterloh, Lebensbedingungen (wie Anm. 68), S. 172.

78 Posta, Terror (wie Anm. 54), S. 205.

79 So „hatte sich im allgemeinen die Lage der sowjetischen Kriegsgefangenen in den deutschen Lagern [...] im Laufe des Jahres 1942 insofern geringfügig verbessert, als nach grundsätzlichen Entscheidungen der nationalsozialistischen Führung, sie nun 
Zweck der Kriegsgefangenenunterbringung wurden die Lager der Ostarbeiter, die an sich schon umzäunt und von minimalem hygienischen Standard waren, nochmals nach den Vorschriften der Abwehrstellen der Wehrmacht ${ }^{80}$ umgebaut, häufig wurden auch Ställe u. ä. genutzt. Ab Ende 1943 waren Kriegsgefangene nicht mehr nur im ehemaligen Messingwerk Lugau - einem temporären „Ostarbeiterlager“ sondern auch in Gebäuden des 1935 aufgegebenen Vertrauensschachtes und in Holzbaracken auf dem Betriebsgelände des Gottes-Segen-Schachtes untergebracht. ${ }^{81}$ Die sowjetischen Kriegsgefangenen wurden dabei von den westeuropäischen Gefangenen isoliert und besonders rabiater Behandlung unterworfen. ${ }^{82}$

Generell war der Umgang der deutschen Belegschaft mit den Gefangenen ein sehr zwiespältiger und einerseits durch Rohheitsakte, wie Prügeln und Antreiben, ${ }^{83}$ andererseits aber auch durch Solidarität bestimmt. Dass die deutschen Belegschaftsangehörigen diesen Menschen (wie auch schon bei den Ostarbeitern) im Einzelfall Essen oder angerauchte Zigaretten überließen, wurde von der Werksleitung auf Gottes Segen streng kritisiert ${ }^{84}$ und durch den Bau getrennter Essensräume unterbunden. Rohheitsakte gegen diese Kriegsgefangenengruppe hingegen, wie Schläge oder Beschimpfungen, wurden in der Regel nicht verfolgt und stillschweigend geduldet. ${ }^{85}$ Die Gewerkschaftsleitungen teilten in der Hauptsache die NS-Rassenideologie und unterstellten den ausgezehrten, zum Teil von Ruhr und Fleckfieber betroffenen Kriegsgefangenen Schmutzigkeit, Faulheit und Heimtücke. ${ }^{86}$ Sie ersetzten die „Abgänge“ (bis zu 30 \% im ersten Jahr) aus den Stamm-

doch als Arbeitskräfte einzusetzen, von deutscher Seite erstmalig ein gewisses Interesse an ihrem Überleben gewährleistet war"; ebd., S. 206.

80 Osterloh hebt hervor, dass erstens nahezu alle vom Deutschen Reich gemachten Kriegsgefangenen der Wehrmacht unterstanden und dass diese darüber hinaus diese Gefangenen nach ihrer Stellung in der NS-Rassenlehre differenziert behandelte; vgl. OsterLOH, Lebensbedingungen (wie Anm. 68), S. 161.

81 Vgl. BA Freiberg, Bestand 40110-1 - 171 .

82 Zum Beispiel galt nach Osterloh, Lebensbedingungen (wie Anm. 68), S. 164, laut dem „Merkblatt für die Bewachung sowjetischer Kriegsgefangener“ vom September 1941 der Schießbefehl (vgl. zur Durchführung auf Gottes Segen: SEIdel/Tenfelde, Zwangsarbeit (wie Anm. 67), S. 885) und erhielten russische Gefangene besonders schlechte Nahrung und Bekleidung (größtenteils aus Restbeständen; vgl. zum „Russenbrot“ Osterloh, Lebensbedingungen (wie Anm. 68), S. 168).

Vgl. Posta, Terror (wie Anm. 54), S. 212 f.

84 „In einem Appell der Betriebsleitung wurde die Belegschaft aufgefordert, jegliche zwischenmenschlichen Kontakte jenseits der unvermeidbaren Kommunikation bei der Arbeit zu unterlassen“; Posta, Terror (wie Anm. 54), S. 202.

85 Vgl. Seidel/Tenfelde, Zwangsarbeit (wie Anm. 67), S. 788.

86 Einige charakteristische entwertende Aussagen der Grubenleitung von Gottes Segen über die sowjetischen Kriegsgefangenen lauteten unter anderem: Im Durchschnitt ist das Gewicht des einzelnen Russen in der Zeit von Okt. 43 bis Mitte Februar 1944 um 7,1 kg zurückgegangen. (Kurt Mauersberger, Betriebsplan 1944, 17. Abschnitt: Kurzer zusammenfassender Betriebsrückblick auf 1943 und betriebliche Vorschau für 1944 (B), S. 9. Die Gewichtsverluste waren im Einzelnen deutlich höher, wie die entsprechende Statistik von Gottes Segen vom 11. März 1944 zeigt; vgl. SeIdel/Tenfelde, Zwangsarbeit (wie Anm. 67), S. 676.) Die Durchschnittleistung des Russen entsprach etwa 
lagern IV F Hartmannsdorf und IV B Mühlberg. ${ }^{87}$ Im Jahr 1943, als sich der Einsatz von Zwangsarbeitern im deutschen Steinkohlenbergbau verstetigt hatte, wurden auf Ministeriumsebene spezielle Anweisungen über den Einsatz der „Ausländer“ im Steinkohlenbergbau erarbeitet ${ }^{88}$ und am 1 . November durch die „Anordnung zur Sicherung der Ordnung in den Betrieben“ des „Generalbevollmächtigten für den Arbeitseinsatz“ Fritz Sauckel ergänzt, wodurch auf der deutschen Seite der Belegschaft die Arbeitnehmermitbestimmung stark eingeschränkt wurde und die Betriebsleitung verpflichtet (und ermächtigt) wurde, die Arbeitsdisziplin im Betriebe dauernd zu überwachen und Verstößen [...] entgegenzutreten. ${ }^{89}$

Die komplette Erfassung und Mobilisierung der erwerbstätigen Bevölkerung durch das Reichsministerium für Rüstung und Kriegsproduktion sowie die der Zwangsarbeiter durch den „Generalbevollmächtigten für den Arbeitseinsatz“ ist demzufolge als parallele Strategie der Ausnutzung menschlicher Arbeitskraft zu sehen. ${ }^{90}$ Diese Ausbeutung ihrer Arbeitskraft traf dabei zwar Kriegs- und Strafgefangene besonders hart, doch machte sie in abgeschwächter Form auch vor der ansässigen Belegschaft ${ }^{91}$ nicht halt, die im schlimmsten Fall ihren relativ geschützten Status verlieren und an die Front einberufen werden konnte. Der Umfang von zwangsweise geleisteter Arbeit im Lugau-Oelsnitzer Steinkohlenbergbau war in der Endphase des Dritten Reiches so erheblich, ${ }^{92}$ dass sich beispielsweise durch den Abgang von Kriegsgefangenen und Ostarbeitern die angelegte Gruben-Beleg-

50-60\% gemessen an der Leistung eines deutschen Arbeiters (MaUersberger, Betriebsplan (wie Anm. 86), S. 9 f.). Der „Russe an sich [ist] willig, leicht zu behandeln, muß immer unter Druck stehen, sonst disziplinlos, faul und dreckig, betrügerisch [...]. Keine Kameradschaft untereinander (ebd., S. 11).

87 Vgl. Posta, Terror (wie Anm. 54), S. 207.

88 Vgl. ebd.

89 Bekanntmachung betr. Anordnung des Generalbevollmächtigten für den Arbeitseinsatz zur Sicherung der Ordnung in den Betrieben vom 1. November 1943; BA Freiberg, Bestand $40110-1-18$.

90 Vgl. auch Oelsnitzer Volksbote 80 (1943), 24, S. 1.

91 „In den Kriegsjahren war der Bergbau eine der Schlüsselindustrien. Die Kohle war eine der wichtigsten Energiequellen. [...] Die Arbeitslast für die Bergleute nahm immer mehr zu und die Verschleißerscheinungen bei den Bergleuten [...] erreichten einen Grad, der mit körperlicher und psychischer Erschöpfung bezeichnet werden mußte“; MenzeL, Bergbaumedizin (wie Anm. 22), S. 277.

92 Ein Schreiben des Gottes-Segen-Betriebsführers Steinmayer aus dem Jahre 1944 an das Arbeitsamt Lugau/Erzgebirge (Meldung vom 31. August 1944 über Bedarf an Arbeitskräften; BA Freiberg, Bestand $40110-1$-18) trug einen Bedarf von knapp 600 Kriegsgefangenen vor. Für 1943 wurde ein Bedarf von 821 Kriegsgefangenen ausgewiesen, angelegt waren letztendlich 706 Kriegsgefangene und 96 Ostarbeiter. 
schaft im sächsischen Steinkohlenbergbau von 14.733 Arbeitern im Jahr 1944 auf 10.724 im Jahr 1945 verringerte. ${ }^{93}$

Die nachfolgenden beiden Übersichten zeigen die zahlenmäßige Dimension des Einsatzes von Ostarbeitern und Kriegsgefangenen in den drei größten sächsischen Steinkohlenwerken:

\begin{tabular}{|l|l|l|l|l|l|l|l|l|l|}
\hline & \multicolumn{3}{|c|}{ GW Deutschland } & \multicolumn{3}{c|}{ GW Gottes Segen } & \multicolumn{3}{c|}{ GW Morgenstern } \\
\hline $\begin{array}{l}\text { Monat } \\
\text { /Jahr }\end{array}$ & $\begin{array}{l}\text { russ. } \\
\text { Kriegs- } \\
\text { gef. }\end{array}$ & $\begin{array}{l}\text { \#st- } \\
\text { arbei- } \\
\text { ter“ }\end{array}$ & $\begin{array}{l}\text { Engl. } \\
\text { franz. } \\
\text { Kriegs- } \\
\text { gef. }\end{array}$ & $\begin{array}{l}\text { russ. } \\
\text { Kriegs- } \\
\text { gef. }\end{array}$ & $\begin{array}{l}\text { Ost- } \\
\text { arbei- } \\
\text { ter“ }\end{array}$ & $\begin{array}{l}\text { Engl. } \\
\text { u. franz. } \\
\text { Kriegs- } \\
\text { gef. }\end{array}$ & $\begin{array}{l}\text { Kuss. } \\
\text { Kriegs- } \\
\text { gef. }\end{array}$ & $\begin{array}{l}\text { Ost- } \\
\text { arbei- } \\
\text { ter“ }\end{array}$ & $\begin{array}{l}\text { Engl. } \\
\text { u. franz. } \\
\text { Kriegs- } \\
\text { gef. }\end{array}$ \\
\hline $6 / 42$ & 0 & 75 & 0 & 0 & 0 & k. A. & 0 & 75 & 0 \\
\hline $12 / 42$ & 262 & 0 & 0 & k. A. & 0 & 13 & 260 & 0 & 8 \\
\hline $6 / 43$ & 386 & 0 & 0 & 396 & 0 & k. A. & 383 & 0 & 8 \\
\hline $12 / 43$ & k. A. & k. A. & 0 & 494 & 0 & 217 & 648 & 0 & 0 \\
\hline $6 / 44$ & 323 & k. A. & 0 & 493 & 0 & 171 & 665 & 0 & 0 \\
\hline $12 / 44$ & 675 & k. A. & 0 & 839 & 0 & 163 & 850 & 0 & 0 \\
\hline
\end{tabular}

Tab. 3: Die absoluten Zablen angelegter Fremdarbeiter. ${ }^{94}$

\begin{tabular}{|l|l|l|l|l|l|l|}
\hline & \multicolumn{2}{|c|}{ GW Deutschland } & \multicolumn{2}{c|}{ GW Gottes Segen } & \multicolumn{2}{c|}{ GW Morgenstern } \\
\hline Jahr & Deutsche & Ausländer & Deutsche & Ausländer & Deutsche & Ausländer \\
\hline 1939 & 3.194 & k. A. & 3.598 & k. A. & 3.772 & k. A. \\
\hline 1940 & 3.138 & 3 & 3.582 & k. A. & 3.759 & k. A. \\
\hline 1941 & 3.046 & 11 & 3.413 & 10 & 3.710 & k. A. \\
\hline 1942 & 3.039 & 118 & 3.234 & 120 & 3.680 & 129 \\
\hline 1943 & 2.828 & 442 & 3.145 & 474 & 3.175 & 476 \\
\hline 1944 & 2.718 & 602 & 3.008 & 729 & 2.683 & 751 \\
\hline
\end{tabular}

Tab. 4: Die nach In- und Ausländern differenzierte durchschnittliche Jabresbelegschaft. ${ }^{95}$

Zusammenfassend kann bemerkt werden, dass im westsächsischen Steinkohlenbergbau insgesamt mehrere Tausend Kriegsgefangene zwangsverpflichtet wurden, wovon der größte Teil der sowjetischen Kriegsgefangenen unter katastrophalen Bedingungen leben und arbeiten musste. Es kam bezüglich der einzelnen Kontingente von Ostarbeitern, Gefangenen der Ostfront und alliierten Kriegsgefangenen

93 Vgl. BA Freiberg, Bestand 40098 - 1 - 664.

94 Quelle: Posta, Terror (wie Anm. 54), S. 220.

95 Quelle: ebd., S. 221. 
zum Teil zu einer „Verschiebung“ der Personen zwischen den Gewerkschaften. ${ }^{96}$ Zudem wechselte die Zuständigkeit über Tage, d. h. außerhalb der Arbeitsorte, je nach dem Status als „Kriegsgefangener“ oder „Zivilarbeiter“ zwischen Wehrmacht und Gestapo. ${ }^{97}$ Ab Ende 1944 sorgte die desaströse wirtschaftliche und militärische Lage des Dritten Reiches für die erneute Zuweisung von 1.200 russischen Kriegsgefangenen aus dem Lager Mühlberg in die westsächsischen Steinkohlenbetriebe, ${ }^{98}$ um mehr deutsche Bergleute an die Wehrmacht und den Volkssturm abstellen zu können. Andererseits wurden sowjetische Zwangsarbeiter auch teilweise ungeordnet $\mathrm{zu}$ anderen kriegswichtigen Arbeiten abgezogen oder in antisowjetische Freiwilligenverbände an der Ostfront überstellt, ${ }^{99}$ sodass von einem organisatorischen Chaos und von zum Teil gegensätzlichen Interessenlagen auf Seiten der deutschen Profiteure der Zwangsarbeit (Betriebe, Wehrmacht) gesprochen werden kann.

Ohne die politischen Systeme des Dritten Reiches und der DDR hiermit gleichzusetzen, ist an dieser Stelle auf die Tatsache aufmerksam zu machen, dass auch nach 1945 in signifikantem Umfang Gefangene im westsächsischen Steinkohlenbergbau eingesetzt waren, wobei beispielsweise die in Oelsnitz angelegten Strafgefangenen in Baracken in der Nähe des Friedrich-Engels-Schachtes untergebracht waren. Generell bestand die unterstellte Gefahr der Fraternisierung der Belegschaft mit den (zum Teil aus politischen Gründen oder wegen sogenannter Devisenvergehen) in den Bergbau abkommandierten Personen in noch viel stärkerem Maße als zur Zeit des Dritten Reiches. ${ }^{100}$

\begin{tabular}{|l|l|l|l|l|l|l|}
\hline Schachtanlage & $\begin{array}{l}\text { Karl- } \\
\text { Liebknecht- } \\
\text { Schacht }\end{array}$ & $\begin{array}{l}\text { Rudolf- } \\
\text { Breitscheid- } \\
\text { Schacht }\end{array}$ & $\begin{array}{l}\text { Deutsch- } \\
\text { land- } \\
\text { schacht }\end{array}$ & $\begin{array}{l}\text { Martin } \\
\text { Hoop } \\
\text { III }\end{array}$ & $\begin{array}{l}\text { Martin } \\
\text { Hoop } \\
\text { IV }\end{array}$ & $\begin{array}{l}\text { Karl } \\
\text { Marx } \\
\text { I }\end{array}$ \\
\hline $\begin{array}{l}\text { Angelegte } \\
\begin{array}{l}\text { Angefahrene } \\
\text { (Grube) }\end{array}\end{array}$ & 194 & 87 & 270 & $/$ & $/$ & $/$ \\
81 & 241 & 44 & 44 & 81 \\
\hline
\end{tabular}

Tab. 5: Angelegte Strafgefangene in den sächsischen Steinkoblenbetrieben im Jabr 1951. ${ }^{101}$

\footnotetext{
96 Vgl. ebd., S. 214 f.

97 Vgl. Seidel/Tenfelde, Zwangsarbeit (wie Anm. 67), S. 318.

98 Posta, Terror (wie Anm. 54), S. 218.

99 Vgl. ebd.

100 Vgl. BA Freiberg, Bestand $40098-1-238$.

101 Quelle: BA Freiberg, Bestand 40098-1-722. Die Angaben beziehen sich auf den Stand vom 1. März 1951.
} 


\section{Die Politische Aufladung in der Endphase des westsächsischen Steinkoblenbergbaus}

Ab Mitte April 1945 war infolge der Kampfhandlungen um Lugau und Oelsnitz kein geordneter Grubenbetrieb mehr möglich. ${ }^{102}$ Darüber hinaus gab es nach Kriegsende eine Vielzahl von Bewegungseinschränkungen durch die Anordnungen der Besatzungsmacht UdSSR und durch die vor dem Gebietstausch räumlich noch sehr nahe Grenze zwischen sowjetisch und amerikanisch besetzter Zone. Hinsichtlich der Material-, Rohstoff- und Ersatzteilbeschaffung gehörten eine ständige Mangelwirtschaft ${ }^{103}$ und das fortgesetzte Ersuchen um Material und um die Genehmigung von Transportfahrten zum Alltag. ${ }^{104}$

Der Grubenbetrieb ${ }^{105}$ lief jedoch rasch wieder an und nach einem kurzzeitigen Rückgang der Fördermengen und Belegschaftszahlen, welcher sich in den Betriebsstatistiken von 1945 bis 1947 nachvollziehen lässt, wurde die Steinkohlenproduktion vonseiten der sowjetischen und der ostdeutschen Administration forciert. Die Energieträgergewinnung hatte in der rohstoffarmen SBZ und späteren DDR eine große wirtschaftliche und politische Bedeutung, sodass generell der Bergmannsberuf neben einer (vor allem in den Betrieben der SDAG WISMUT) vergleichsweise hohen Bezahlung auch großes gesellschaftliches Ansehen genoss und jährlich am „Tag des Bergmanns“ mit Aufmärschen und Versammlungen in den Bergbauorten gewürdigt wurde.

Die organisatorischen Strukturen im sächsischen Steinkohlenbergbau wurden unter Federführung der Sowjetischen Militäradministration in Deutschland grundlegend geändert, darüber hinaus wurden zum Teil ehemals politisch Verfolgte, wie beispielsweise im Betrieb Deutschland das KPD-Mitglied Willy Mehlhorn, als Werkleiter eingesetzt. Am 5. Januar 1946 wurden die Gewerkschaften Deutschland und Gottes Segen zunächst vorübergehend mit den anderen sächsischen Steinkohlenproduzenten zur landeseigenen Sächsischen Steinkohlenwerke $\mathrm{GmbH}$ zusammengefasst, um nach zwischenzeitlicher erneuter Ausgliederung

102 Vgl. Vogel, Steinkohlenrevier (wie Anm. 2), S. 46; BA Freiberg, Bestand 40110 $1-24$.

103 Die Steinkohlenförderung stagnierte in den späten 1940er-Jahren vor allem aus folgenden Gründen: unzureichende Regeneration durch mangelhafte Ernährung (teilweise nur $50 \%$ der medizinisch notwendigen Kalorienzufuhr) und zu wenige arbeitsfreie Tage (1947: 303 Werktage + 27 Sonntage); abgenutzte und häufig reparierte Betriebsmittel, hohe Ausfallquote, Verletzungsrisiko; Materialknappheit bei Zulieferern aufgrund der Reparationsverpflichtungen; fehlender Kollektivgeist durch mangelnde Bergbautauglichkeit vieler Neuzugänge und durch hohe Fluktuation (unter anderem Abwerbung durch WISMUT); logistische Probleme beim Transport auswärtiger Arbeiter (Bahn/Fahrrad); geologische Schwierigkeiten und Abnahme der Flözqualität. Vgl. BA Freiberg, Bestand $40098-1-805$.

104 Vgl. BA Freiberg, Bestand $40110-1-1146$.

105 Im Lugau-Oelsnitzer Revier nur noch in den Gewerkschaften Deutschland und Gottes Segen, der Gersdorfer Steinkohlenbauverein ging 1944 aus wirtschaftlichen Gründen (mangelnde Erlöse) in Verbindung mit natürlichen Ursachen (Wassereinbruch) ein. 
der Gewerkschaft Deutschland (diese firmierte ab Juli 1952 als VEB Steinkohlenwerk Deutschland) schließlich im Jahre 1960 als VEB Steinkohlenwerk Oelsnitz ${ }^{106}$ die letzte Etappe der Steinkohlenförderung im Lugau-Oelsnitzer Revier zu beginnen. Die Sächsische Steinkohlenwerke GmbH wurde ihrerseits in einem nächsten Zentralisationsschritt der „Industrieverwaltung 1 (Steinkohle)“107 unterstellt und bestand in dieser Form bis 1948. Im Juli 1948 kam es dann zur Gründung der „Vereinigung Volkseigener Betriebe (VVB) Steinkohle Zwickau“,108 welche bis zum Jahr 1977 bestand. 109

Von Anfang an wirkte ein starker wirtschaftlicher und politischer Druck zur Erhöhung der Fördermengen auf die Steinkohlenproduktion, da, wie schon erwähnt wurde, das vom Krieg ausgezehrte Land unter einem Energie- und Ressourcenmangel litt und außerdem stark durch die Reparationsleistungen an die UdSSR beansprucht wurde. Zudem waren auch die bekannten abbauwürdigen Kohlenvorräte im Lugau-Oelsnitzer Revier deutlich zurückgegangen, wie die diesbezüglichen Verhandlungen zwischen den Werken Gottes Segen und Deutschland belegen, die bereits unter dem Gesichtspunkt der wirtschaftlichen Ausbeutung von „Restflächen“ (Sicherheitspfeiler, aufgelassene Schächte, Grenzflächen u. ä.) und einer sich abzeichnenden Erschöpfung der Kohlenvorräte geführt wurden. ${ }^{110}$ Darüber hinaus trat ab 1949/50 die SDAG Wismut als Konkurrent um die Arbeitskräfte in Erscheinung. Die Löhne im benachbarten Uranabbaugebiet waren teilweise doppelt so hoch wie für adäquate Tätigkeiten in der Steinkohlengewinnung.

Größte Bedeutung für den Abschluss der westsächsischen Steinkohlenproduktion hatten der Beschluss des Präsidiums des Ministerrates der DDR vom 21. Dezember 1967 zur schrittweisen Einstellung der Steinkohlenförderung (Verfügung 11/67) im Lugau-Oelsnitzer Gebiet und der damit korrespondierende Beschluss vom 12. August 1970 für die Region Zwickau. ${ }^{111}$ Mit diesen Direktiven

106 Aus Deutschland war, wie schon erwähnt wurde, im Juli 1952 der VEB Steinkohlenwerk Deutschland geworden, Gottes Segen trat als VEB Steinkohlenwerk Karl Liebknecht in die Verbindung ein.

107 Bis 1949 existierte ein „(Direktorat der) Verwaltung der Kohlenindustrie des Landes Sachsen“, mit einer "Abteilung Steinkohle“ in Zwickau; vgl. BA Freiberg, Bestand $40098-1-879$.

108 Die VVB Steinkohle Zwickau bestand neben den Zwickauer (Karl Marx, Martin Hoop und August Bebel) und Oelsnitzer Steinkohlenbetrieben (VEB Steinkohlenwerk Deutschland und VEB Steinkohlenwerk Karl Liebknecht; per VVB-Anweisung von Hauptdirektor Köhler vom 7. November 1959 mit Wirkung zum 1. Januar 1960 zum VEB Steinkohlenwerk Oelsnitz/Erzgebirge vereinigt) aus dem VEB Zentralwerkstatt Niederwürschnitz, dem VEB Steinkohlenwerk Plötz (in Sachsen-Anhalt) und dem VEB Steinkohle Willi Agatz in Freital. Der Generaldirektor Scheitler war vormals der Werkdirektor des VEB Steinkohlenwerk Martin Hoop in Zwickau; vgl. BA Freiberg, Bestand $40098-1-238$.

109 Vgl. Erb/Harring, Aus der Tiefe ans Licht (wie Anm. 3), S. 47.

110 Vgl. BA Freiberg, Bestand $40109-1-439$.

111 Vgl. Wolfgang Scheitler, Geschäftsbericht der VVB Steinkohle, Zwickau für das Jahr 1970, Zwickau 1971 (BA Freiberg, Bestand 40098 - 1 - 411, S. 67). 
wurde das definitive Ende der sächsischen Steinkohlenförderung nach einer zumindest im Zwickauer Raum recht langen bergmännischen Tradition und nach fast 20 Jahren der Subventionierung ${ }^{112}$ durch die DDR-Staatsführung besiegelt. Die Raub- und Verwahrungsarbeiten nahmen in beiden Revieren noch wenige Jahre und eine geringe Zahl von Arbeitskräften in Anspruch; seit Mitte der 1970erJahre kann vom definitiven Ende der Steinkohlenproduktion in der Region Lugau-Oelsnitz (letzte Förderschicht 1971) und seit 1980/81 auch in Zwickau (letzte Förderschicht 1977) gesprochen werden.

Auch wenn es durchaus noch Anstrengungen gab, die Förderleistung zu maximieren, und auch wenn in den frühen 1960er-Jahren ein umfangreiches Prämiensystem die Arbeitsmotivation unterstützen sollte, lag der Fokus der Planungen ab den 1960er-Jahren eindeutig auf dem Aufbau von Nachfolgebetrieben und der Qualifikation und Umsetzung der bisher in der Steinkohlenproduktion beschäftigten Arbeitskräfte.

1. Politische Aufladung der Förderung, Wettbewerbs- und Prämienwesen Vonseiten der sowjetischen und später der ostdeutschen Administration gab es ab 1946/47 Bestrebungen, mit Adolf Hennecke (Neuoelsnitz) und Franz Franik (Zwickau) Vorbildfiguren zu etablieren, die über die Aktivisten- bzw. später die Franikbewegung einen erheblichen Druck auf die Normerfüllung und den Verbrauch technischer Hilfsmittel, auf Krankmeldungen, Feierschichten etc. ausübten. ${ }^{113}$

Zum Zeitpunkt der minutiös geplanten und dokumentierten Hennecke-Aktion (knapp 400-prozentige Normerfüllung) im Oktober 1948 wirkten jedoch noch viele der oben erwähnten Hemmnisse, wie ungenügende Ernährung, Wohnungsknappheit oder fehlendes Material, auf die Arbeitssituation ein. Die Unfallstatistik für das Jahr 1949 zeigt die problematischen Arbeitsumstände unter Tage:

\begin{tabular}{|l|l|l|l|l|l|l|l|l|l|}
\hline Werk & \multicolumn{3}{|c|}{ Karl Liebknecht } & \multicolumn{3}{c|}{ Deutschland } & \multicolumn{3}{c|}{$\Sigma$ VVB Steinkohle } \\
\hline Monat & tödl. & schwer & leicht & tödl. & schwer & leicht & tödl. & schwer & leicht \\
\hline Januar & $/$ & 12 & 87 & $/$ & $/$ & 87 & 6 & 37 & 418 \\
\hline Februar & 1 & 5 & 119 & $/$ & 1 & 81 & 3 & 29 & 525 \\
\hline März & 1 & 5 & 111 & $/$ & 6 & 106 & 1 & 32 & 570 \\
\hline
\end{tabular}

112 Der starke Produktionsrückgang ist ausschließlich auf die Arbeitskräftesituation zurückzufübren. Die kritischste Bilanzlücke unserer Pläne [...] lag in dem Feblen von Arbeitskräften. Eine bemerkenswerte Steigerung der Arbeitsproduktivität, um damit diese Bilanzlücke zu schließen, konnte nicht erreicht werden. Die Arbeitsproduktivität liegt so niedrig, daß das Werk nicht in der Lage ist, die anfallenden Selbstkosten aus den Erlösen zu decken; Politisch-ökonomische Konzeption zur Durchführung der Plandiskussion 1967 und zur Rechenschaftslegung zum BKV 1. Halbjahr 1966, S. 10, in: VEB Steinkohle Oelsnitz/Erzgebirge. Betriebsplan 1967, Teil 1 (BA Freiberg, Bestand $40120-1-315)$.

113 Vgl. BA Freiberg, Bestand 40116 - 1 - 375; und Franz Franik, Die Franikbewegung und der sozialistische Wettbewerb im Bergbau, Berlin 1953, S. 13 f. 


\begin{tabular}{|l|l|l|l|l|l|l|l|l|l|}
\hline April & 1 & 5 & 85 & $/$ & 8 & 90 & 1 & 37 & 471 \\
\hline Mai & 1 & 11 & 119 & $/$ & 3 & 105 & 1 & 55 & 490 \\
\hline Juni & $/$ & 7 & 126 & $/$ & 3 & 114 & 2 & 26 & 554 \\
\hline Juli & $/$ & 9 & 105 & $/$ & 8 & 102 & $/$ & 38 & 478 \\
\hline August & 1 & 10 & 102 & $/$ & 5 & 107 & 1 & 33 & 508 \\
\hline September & $/$ & 11 & 93 & $/$ & 6 & 87 & $/$ & 51 & 466 \\
\hline Oktober & 1 & 13 & 83 & $/$ & 5 & 87 & 1 & 42 & 407 \\
\hline November & 1 & 4 & 69 & $/$ & 7 & 56 & 2 & 29 & 365 \\
\hline Dezember & 1 & 4 & 99 & $/$ & 3 & 76 & 1 & 27 & 425 \\
\hline
\end{tabular}

Tab. 6: Unfallstatistik der VVB Steinkoble für das Jabr 1949.114

Eine Lösung der diffizilen Konfliktlage zwischen der Materialknappheit und der physischen Erschöpfung vieler Grubenarbeiter in der Nachkriegszeit sowie den hohen Arbeitsanforderungen und politisch motivierten Planzahlen wurde unter anderem durch republikweit initiierte Wettbewerbe angestrebt. Das Wettbewerbswesen wurde durch die 1946 gegründete Sozialistische Einheitspartei Deutschlands ${ }^{115}$ unter politisch-ideologischer Konnotation und unter Einbeziehung vieler Medien,116 wie Wandzeitung, Betriebsfunk, Film und Tageszeitungen, in allen Branchen organisiert. Neben wöchentlichen und vierteljährlichen Vergleichen wurden die Kumpel zum Teil auch während der Schicht über ihren Tages-Produktionsstand informiert und mit den jeweils besten und schlechtesten Leistungen öffentlich herausgestellt, sodass ein gewisser psychischer Druck zur Planerfüllung117 entstand. Dieser Druck - auch durch die sogenannten Kubiker wie Franik

114 Quelle: VVB Steinkohle Zwickau. Jahresbericht an die HA Kohle vom 7. Dezember 1949, Anlage 11 (BA Freiberg, Bestand 40098 - 1 - 823).

115 In einer Erfassung der Organisationsmitgliedschaft der Betriebsangehörigen (ohne Lehrlinge) des Karl-Liebknecht-Werkes vom Jahresende 1950 wird sichtbar, dass alle 339 Angestellten Mitglieder des FDGB und 130 Personen (= 38,35 \%) Mitglieder der SED waren. Der Anteil ehemaliger NSDAP-Mitglieder betrug 83 Personen $(=24,48$ $\%)$. Bei den 5138 Produktions- und Produktionsgrundarbeitern - ebenfalls alle Mitglieder des FDGB - ergab sich ein Anteil von 1270 (= 24,71 \%) Mitgliedern der SED und $115(=1,95 \%)$ ehemaligen NSDAP-Mitgliedern; vgl. BA Freiberg, Bestand 40118 $-778$.

116 Im Revier neben der Freien Presse und dem Neuen Deutschland ab 1948/49 unter anderem Die Grubenlampe. Mitteilungen der VVB Steinkohle Sachsen (herausgegeben durch die jeweiligen SED-Betriebsgruppen), 1952 Die Brücke. Mitteilungsblatt der Zweijahresschule für den Steinkohlenbergbau in Oelsnitz für die Eltern und Erzieher ihrer Lehrlinge (ebenfalls von Betriebsparteiorganisationen herausgegeben), 1953 Echo des Werkes. Betriebszeitung für die Belegschaft des Werkes Deutschland und Dr Bargmaa; vgl. BA Freiberg, Bestand $40098-1-404$.

117 Die Abteilungen verpflichteten sich (teilweise namentlich), das Plansoll um einige Prozent überzuerfüllen (vgl. Franik, Franikbewegung (wie Anm. 113), S. 35 f.), Material zu sparen etc. Es gab mehrere Normerhöhungen (vgl. "technisch begründete Normen“) und sogar Lohnabzüge bei Unterschreitung des Plansolls. Selbst für den Krankenstand gab es ein Höchst-Soll, bei dessen Überschreitung zum Teil Schlagworte wie „Unkameradschaftlichkeit" und "Schmarotzertum" in Gebrauch kamen; vgl. BA Freiberg, Bestand 40116 - 1-245. 
in Zwickau - wurde teilweise so hoch, dass es zu Konflikten zwischen FDGBFunktionären und den Normeningenieuren kam, da der FDGB die „technisch begründeten Normen" in einigen Bereichen für überhöht hielt.118

Während über das Gedinge hinaus in der Zeit vor 1945 keine explizite Vergütung einer Planübererfüllung erfolgte, wurde ab 1946 auch die Einführung von Prämien ${ }^{119}$ erwogen, um die Produktionsleistung zu erhöhen, schließlich hatte es auf Grund der Normerhöhungen und wegen mangelnden materiellen Anreizen im Steinkohlenbergbau Ende der 1940er- und Anfang der 1950er-Jahre viele Abgänge von den Schächten gegeben. Der VEB Karl Liebknecht verzeichnete im Jahr 1954 bei 1.645 Zugängen 2.090 Abgänge, bei Deutschland erfolgten 675 Neuanlegungen bei gleichzeitig 1.340 Abgängen.120 Nach der Einführung der „Zyklusarbeit“ in den 1950er-Jahren in den Betrieben des VVB Steinkohle wurden mit den „Grundsätze[n] für die Gewährung von Zyklusprämien“121 und über das Deputatwesen signifikante materielle Anreize für den uneingeschränkten Einsatz der eigenen Arbeitskraft in Aussicht gestellt. Das Ziel höherer Produktionszahlen wurde also durch eine politische Aufladung der Arbeit - und gleichzeitig durch materielle Anreize zur Übererfüllung auf allen Hierarchieebenen verfolgt. ${ }^{122}$ Neben dem sozialistischen Wettbewerb wurde im Hinblick auf die Steinkohlenproduktion auch die Weltpolitik zur Bewusstseinsbildung und Leistungssteigerung bemüht, denn es gab im RGW eine Ständige Kommission Kohle, ${ }^{123}$ in der der zuständige Minister Bericht zu erstatten hatte.124

Seit 1947 wurden neben den Jahresprämien (von ca. 60 \% eines Monatslohns) auch wieder Deputatmarken für den Bezug von Kohlen zum stark vergünstigten Preis ausgegeben. Sie fungierten in ihrer Eigenschaft als Lohn- bzw. Honorarbestandteil für Handwerker (Schlosser, Schuster, Gärtner, Gerichtsschreiber) oder Dienstleister zum Teil als inoffizielle „Ersatzwährung“ (und nahmen in gewisser Weise den späteren inoffiziellen Handel mit den Bezugsmarken für akzisefreien Schnaps vorweg, der auch im benachbarten WISMUT-Gebiet aufkam).

Neben diesen unmittelbaren Begleiterscheinungen der Steinkohlenförderung kam es auf der infrastrukturellen Ebene im Revier zu einem umfangreichen Ausbau der bereits seit dem späten 19. Jahrhundert sukzessive angelegten Bergbau-

118 Vgl. BA Freiberg, Bestand $40098-1-722$.

119 Vgl. zum Prämienwesen auch Scheitler, Geschäftsbericht 1971 (wie Anm. 111), S. 91.

120 Vgl. BA Freiberg, Bestand $40116-1-245$.

121 Wolfgang Scheitler, Geschäftsbericht der VVB Steinkohle, Zwickau für das Planjahr 1964, Zwickau 1965 (BA Freiberg 40098 - 1-414, S. 12; Bestand $40098-1$ - 238).

122 Vgl. BA Freiberg, Bestand $40098-1-238$.

123 Vgl. BA Freiberg, Bestand $40098-1-35$.

124 Übergeordnete Instanz war das Ministerium für Kohle und Energie mit der Hauptverwaltung Steinkohle in Zwickau, im Jahr 1951 wechselte die Zuständigkeit zum Ministerium für Schwerindustrie, Hauptverwaltung Kohle in Berlin. Zum Vergleich: Im Jahr 1967 wurden in der UdSSR in 4834 „Gewinnungsbetriebspunkten“ 444 Mio. t Steinkohle und 143,4 Mio. t Braunkohle gefördert; Die sozialistische Rekonstruktion. Informationen für den Steinkohlenbergbau der DDR 1 (1967), S. 118. 
siedlungen ${ }^{125}$ (beispielsweise im sogenannten Dichterviertel im Süden von Lugau, im Ortsteil Neuoelsnitz und in der Bebauung im Oelsnitzer Stadtzentrum). Mit Kulturhäusern, Polikliniken, Sportanlagen und Schulen entstand eine vielfältige soziale Infrastruktur, sodass beispielsweise Oelsnitz ab den späten 1960er-Jahren „über 4 zehnklassige polytechnische Oberschulen, eine Betriebsberufsschule, eine Zentralhilfsschule, ein Krankenhaus, eine Poliklinik, mehrere Kindergärten, Kinderkrippen und 2 Sportstadien" verfügte. ${ }^{126}$ Viele Anlagen, wie beispielsweise das Klubhaus des Steinkohlenwerkes in Lugau, die Bergarbeiterpoliklinik Oelsnitz oder das Bergarbeiterkrankenhaus Oelsnitz standen bis 1970 in direkter Rechtsträgerschaft der Steinkohlenbetriebe ${ }^{127}$ und es kann konstatiert werden, dass zumindest bis zum Ende der Förderung - der Raum zwischen Chemnitz und Zwickau sozial und infrastrukturell profitiert hat.

\section{Soziale Folgeeffekte der eingestellten Steinkoblenförderung}

Zum Schluss der überblicksartigen Betrachtung sozialer Begleiterscheinungen der Steinkohlengewinnung im Lugau Oelsnitzer Revier ist kurz auf Folgeeffekte des Einstellungsbeschlusses einzugehen.

Zunächst ergab sich aus dem Beschluss, die hoch subventionierte und unrentable Steinkohlenförderung einzustellen, die Notwendigkeit, mehrere Tausend Arbeitskräfte umzusetzen. Dem letzten Zwickauer Generaldirektor Scheitler zufolge wurden [d]ie Zielstellungen zur Überfübrung der Arbeitskräfte in die festgelegten strukturbestimmenden Betriebe und für die Schaffung neuer Arbeitsplätze [...] 1970 mit Ausnabme geringfügiger und allseitig abgestimmter Änderungen erreicht. Entscheidender Höbepunkt in der Arbeit war die termingemäße Fertigstellung und Inbetriebnabme des ersten Bauabschnittes des Teilbetriebes Karl Liebknecht des Buchungsmaschinenwerkes Karl-Marx-Stadt in Oelsnitz am 1.1.1970.128 Die Quelle nennt in diesem Zusammenhang für 1970 die Zahl von 4.846 umgesetzten Beschäftigten. ${ }^{129} \mathrm{Zu}$ diesen sogenannten strukturbestimmenden Betrieben, die eine adäquate Beschäftigung der freigesetzten Gruben- und Industriebelegschaft sichern sollten und die häufig in unmittelbarer Nachbarschaft der ehemaligen

125 Diese Siedlungen sollten die hygienischen Verhältnisse und die Selbstversorgung der Haushalte mit Nahrung durch Gartenbau und Kleintierhaltung bessern und über die Vergabe von Baumitteln die Bindung an die jeweiligen Steinkohlenbetriebe sichern. Sie können darüber hinaus aber auch als Kontroll- und Normierungsplätze betrachtet werden und bilden in ihren baulichen Typen (Ein- oder Mehrfamilienhäuser mit und ohne Grünland, sogenannte Steigerhäuser etc.) auch die soziale Schichtung im Steinkohlenbergbau ab.

126 Rössler/ZüHLKe, Mülsengrund (wie Anm. 14), S. 121.

127 Vgl. Wolfgang Scheitler, Geschäftsbericht der VVB Steinkohle, Zwickau für das Jahr 1969, Zwickau 1970 (BA Freiberg 40098 - 1 - 412, S. 99).

128 SCheitler, Geschäftsbericht 1971(wie Anm. 111), S. 12.

129 Vgl. ebd., S. 13. 
Förderanlagen installiert wurden, gehörten unter anderem das Gersdorfer „Zweigwerk des VEB Betonleichtbaukombinat Dresden“,130 in Lugau der VEB Edelstahlzieherei (als Zweigbetrieb des Kombinates „8. Mai“ in Freital) und der VEB Wärme- und Sanitärtechnik, in Niederwürschnitz der VEB Textima ${ }^{131}$ sowie in Neuoelsnitz das „Buchungsmaschinenwerk im VEB Robotron“ und das „Plattenwerk des VEB Wilhelm Pieck“.132 Auch wenn Scheitlers Aussagen einen nahtlosen Übergang von der Steinkohlengewinnung in die Nachfolgebetriebe nahe legen, dürfte die Umqualifikations- und Anpassungsphase besonders bei den älteren Arbeitnehmern erhebliche persönliche Ressourcen gebunden haben.

Mit dem tiefgehenden Strukturwandel der Produktionsbetriebe im LugauOelsnitzer Revier - in dem nicht zuletzt auf Betreiben der Gewerke zu Zeiten der Kohlenförderung bis zum Kriegsende vergleichsweise wenige „bergbaufremde“ Industrien angesiedelt wurden - gingen auch städtebaulich und sozialräumlich relevante Folgen der Abkehr von der Steinkohle einher. Der Chemnitz-Zwickauer Raum stellt soziogeografisch gesehen ein Konglomerat von Wohn- und Produktionsstandorten dar, das als durchgehender Bebauungsgürtel ${ }^{133}$ betrachtet werden kann und das in manchen Merkmalen dem Ruhrgebiet ähnelt. Besonders die ehemaligen Kohlenorte bildeten ein sozialräumlich spezifisches Gebiet mit einer hohen Bevölkerungsdichte und gut ausgebauter Infrastruktur. Das Land Sachsen als übergeordnete Einheit hatte seit dem Ende des Zweiten Weltkriegs viele Flüchtlinge und innerdeutsche Wanderer aufgenommen, ${ }^{134}$ was mittelbar dazu führte, dass es ein relativ hohes Durchschnittsalter der Bevölkerung aufwies und dass insbesondere Westsachsen durch seine spezifische ökonomisch-geografische Struktur zu einem „Stiefkind“ der Regionalplanung ${ }^{135}$ wurde, ein Label, unter das (nach dem Ende der Steinkohlenförderung) auch das Lugau-Oelsnitzer Stein-

130 Rössler/ZüHLke, Mülsengrund (wie Anm. 14), S. 76.

131 Vgl. ebd., S. 143.

132 Ebd., S. 146.

133 „Das Ballungszentrum zwischen den Städten Karl-Marx-Stadt und Zwickau ist das bevölkerungsreichste kleinstädtisch-ländliche Siedlungsgebiet der DDR. Auf einem Territorium von etwa $300 \mathrm{~km}^{2}$ lebten 1970 hier über 150000 Menschen. Im mittleren Teil, in dem 10 Gemeinden durch Bebauung unmittelbar zusammenhängen, wird eine Einwohnerdichte von mehr als $900 \mathrm{EW} / \mathrm{km}^{2}$ erreicht. Diese Erscheinung ist in erster Linie eine Folge der Zuwanderung, die der Steinkohlenabbau im Lugau-Oelsnitzer Revier hervorgerufen hatte“; ebd., S. 24.

134 Vgl. Grundmann, Bevölkerungsentwicklung (wie Anm. 29), S. 52 f.

135 „Die in den 70er und 80er Jahren erfolgte Eingliederung vieler Kleinbetriebe in die ,Industrie-Kombinate änderte kaum etwas an ihrer desolaten Situation. Sie blieben ein Randproblem der Planung. Folglich waren auch jene Regionen der DDR, wo die Zahl und der Anteil solcher Betriebe hoch gewesen ist, marginale Gebiete der Territorialplanung. [...] 1967 wurde der Steinkohlenbergbau im Revier Lugau-Oelsnitz eingestellt. Danach wurde das Gebiet zu einer terra incognita der Territorialplanung. [...] Obwohl die Heimatverbundenheit der Bevölkerung traditionell sehr hoch gewesen ist, hatten die zuerst großen (um 1950) dann zwar geringen, aber beständigen Wanderungsverluste [...] verheerende demographische und soziale Konsequenzen. Südwestsachsen [...] wurde zu einer wachsenden Ballung sozialer Probleme“; ebd., S. 120. 
kohlenrevier subsumiert werden kann. Nach Grundmann wurde der Bezirk KarlMarx-Stadt ${ }^{136}$ „[z]um Bezirk mit dem Höchstmaß des Verschleißes der Bausubstanz",137 was maßgeblich durch die oben erwähnte dichte private und gewerbliche Bebauung verursacht wurde, die einen hohen Unterhaltungs- und Sanierungsaufwand bedingte. Hinzu kamen im unmittelbaren Abbaugebiet zum Teil erhebliche Oberflächenabsenkungen von beispielsweise bis zu $19 \mathrm{~m}$ im Oelsnitzer Ortsteil Waldesruhe, die - ebenso wie das nachdringende Grubenwasser noch heute einen signifikanten Investitionsaufwand zur Sicherung der Wohngebiete nach sich ziehen. Das ebenfalls ökologisch schwer belastete UranAbbaugebiet der WISMUT AG liegt in räumlicher Nähe.

Die oben erwähnten demografischen und infrastrukturellen Gegebenheiten warfen nicht nur nach dem Auslaufen der Förderung in den 1970er-Jahren Probleme auf, sondern begünstigten besonders nach dem Strukturwandel der 1990er-Jahre eine Abwanderung der jüngeren Berufstätigen, wodurch beispielsweise im Zeitraum zwischen 1990 und 2004 die Stadt Lugau über 2.100 und die Stadt Oelsnitz über 2.500 Einwohner verloren. ${ }^{138}$

\section{Fazit}

Ein pessimistischer Rückblick auf den Steinkohlenbergbau zwischen Oelsnitz, Niederwürschnitz, Lugau, Gersdorf und Hohndorf ist trotz der geschilderten sozialen Begleitumstände und auch angesichts der ökologischen Folgen nicht angebracht. Im Gegenteil, die ehemaligen Bergbauorte im Revier erwähnen ihre rund 130-jährige Tradition mit Stolz und erinnern vor allem daran, dass die Kohlengewinnung die Gestalt der kleinen vorerzgebirgischen Orte unwiderruflich verändert und in mehrerer Hinsicht die Industrialisierung und Modernisierung begleitet hat.

Nicht nur als Entwicklungsmotor der westsächsischen Industrialisierung und als „Ernährer“ Tausender Familien blieb die bergmännische Tätigkeit in Erinnerung, sondern auch als Grundlage vieler Vereine der Selbst- und gegenseitigen Hilfe, als Beförderer ethnischer und religiöser Vielfalt (die von den Einwanderern besuchten katholischen Gemeinden bestehen zum Teil noch heute) und als direkter oder indirekter Förderer von Sport, ${ }^{139}$ Kultur und Geselligkeit, von Arbeiterbildung und von einem lebhaften Vereinswesen, dass seine Spuren bis in die Gegenwart bewahrt hat.

136 Karl-Marx-Stadt war zwischen 1953 und 1990 der Name der Stadt Chemnitz.

137 Grundmann, Bevölkerungsentwicklung (wie Anm. 29), S. 121.

138 Vgl. Mandy Fischer, Landkreis Stollberg verliert eine Kleinstadt, in: Freie Presse 21. Januar 2005, S. 13.

139 Neben Radsport, Turnen, Schwimmen und Wandern sind vor allem die Ringkämpfer aus Lugau und die Boxsportler aus Oelsnitz zu nennen und die beiden letzteren auch überregional bekannt geworden. 